\title{
The patient's motivation in seeking complementary therapies
}

\author{
P. Ritvo, Ph.D. ${ }^{a, b, c, d, e, f, *}$, J. Irvine, D.Phil. ${ }^{b, c, d}$, J. Katz, Ph.D., ${ }^{b, g}$, A. Matthew, M.A., \\ J. Sacamano, M.D., B.F. Shaw, Ph.D. ${ }^{\mathrm{b}, \mathrm{d}, \mathrm{j}}$ \\ ${ }^{a}$ Division of Preventive Oncology, Cancer Care Ontario, 620 University Avenue, Toronto, Ontario M5G 2L7, Canada \\ ${ }^{\mathrm{b}}$ Department of Public Health Sciences, University of Toronto, Toronto, Ontario, Canada \\ ${ }^{\mathrm{c}}$ Department of Family \& Community Medicine, University of Toronto, Toronto, Ontario, Canada \\ ${ }^{\mathrm{d}}$ Department of Psychiatry, University of Toronto, Toronto, Ontario, Canada \\ ${ }^{\mathrm{D} D i v i s i o n}$ of Epidemiology, Biostatistics and Behavioural Science, Ontario Cancer Institute, 610 University Avenue, Toronto, Ontario, \\ Canada \\ ${ }^{\mathrm{f}}$ Princess Margaret Hospital-University Health Network, 610 University Avenue, Toronto, Ontario, Canada \\ ${ }^{8}$ Department of Anaesthesia, Toronto General Hospital-University Health Network, 200 Elizabeth Street, Toronto, Ontario, Canada \\ ${ }^{\mathrm{h}}$ Department of Psychology, Ontario Institute for Studies in Education, 252 Bloor Street West, Toronto, Ontario, Canada \\ 'Victoria Mental Health Services, Victoria, B.C., Canada \\ ${ }^{j}$ Department of Psychology, Hospital for Sick Children, 555 University Avenue, Toronto, Ontario, Canada
}

\begin{abstract}
The motivations of cancer patients in seeking complementary therapies are, fundamentally, self-healing motivations which, when engaged appropriately, can contribute to the patient's psychological and physical well being. In this paper, we apply a theoretical model, the Risk Adaptation Model, to furthering the clinical understanding of the motivations of cancer patients in seeking complementary therapies. The model identifies six discrete cognitive processes which, in combination, are hypothesized to play a central role in therapy seeking. Emphasis in this model is placed on the patient's need to maintain positive expectancies (optimism) when faced with the risk and uncertainty of cancer. This understanding of complementarytherapy seeking is grounded in the perspective that clinicians must respect the autonomy of cancer patients in their quest for appropriate therapies, and assist rather than direct their process of therapy-seeking. (C) 1999 Elsevier Science Ireland Ltd. All rights reserved.
\end{abstract}

Keywords: Motivation; Therapy-seeking; Complementary therapies

\section{Introduction}

As health care providers serving cancer patients, it

\footnotetext{
*Corresponding author: Tel.: +1-416-971-9800, ext. 1203; fax: $+1-416-596-2428$.
}

is important to understand our patients' motivations in seeking complementary therapies. Fundamentally, these are self-healing motivations which, when engaged appropriately, contribute to the patient's psychological and physical well being. When inappropriately engaged, however, they may lead to self- 
harm. Patients can become so focally motivated by complementary therapies and so reliant on them, that they delay or avoid, or are non-adherent with evidence-based treatment. In this paper, we offer a theoretical model, the Risk Adaptation Model [1,2] (RAM), with the intent of helping clinicians understand the motivations of cancer patients in seeking complementary therapies. With better understandings, we will be more helpful in assisting their decision making.

At the first step of exploring complementary therapy-seeking, we must accept the perspective that, according to our current understanding of placebo effects, virtually any therapy can contribute to "healing", especially if the patient has strong beliefs about its healing properties. Accepting this is part of acknowledging the primacy of the autonomy of patients in relating to their disease and recognizing that they are best served by our assisting this autonomous process. Our assistance, however, is inevitably based in some model, implicit or explicit, of health-seeking. For several years, our group has focussed on the study of health-seeking, particularly in specifying motivational factors. We have assimilated clinical observation and relevant theory into a cognitive-behavioural model, applicable clinically $[1,2]$ and testable experimentally $[3,4]$ that integrates salient components from other cognitive models of health behaviour change [5-7], self-efficacy theory [8], and from the Transtheoretical Model of personal change $[9,10]$. While no model of this type can yet claim definitiveness, a model such as ours can be a useful orienting device, especially when there is concern that the patient's health-seeking process is leading to some form of self harm.

From our model's perspective, health seeking is an active, decision-driven process. As such, the choice of taking or not taking a health-oriented approach is confronted in every situation. Even adopting a passive approach is an active process, cognitively, and, in the right circumstance, passivity is a healthy option. When facing significant health risks, however, patients are rarely passive. Instead, they actively attempt to reduce mortality risks, the risks of escalating disease and distress, and lifestyle limitations, which naturally include illness-related pain, be it physical or psychic in nature.

\section{The model}

Our model encompasses six cognitive processes. These include modifiability, consequentiality, response efficacy, self efficacy, and attention regulation. The sixth cognitive process refers to the influence of expectancies, specifically generalized expectancies such as optimism and pessimism. According to the model, these processes are activated when the patient, in seeking health through complementary therapies, questions how modifiable a current health state is. Given the difficulty of ascertaining this without trial and error, questions follow about the consequences of attempting modification using a particular method. We believe patients, informally or formally, weigh the "pros" and "cons" of attempting versus not attempting specific modifications. This weighing involves judgements of response efficacy, or how effective the method may optimally be and self efficacy, or how effective they may be in using the method. These questions and evaluations evolve as attention is regulated towards addressing them. Attention regulation, another key model component, is defined as the degree of functional attention directed to a given content area. Broadly speaking, patients may seek and find increasingly useful information about complementary therapies or conduct a fragmented inquiry, avoiding specific and even critical questions altogether. Recognizing the potentially infinite combinations of inquiry and disengagement, we must accept that patients will inevitably proceed with or diverge from critical examinations of health information in ways which, although arbitrary and unsystematic to us, may seem "reasonable" to them. Thus pointing out deviations from systematic, critical examination can be an important part of the service we provide, as long as the patient's autonomy in the examination process is respected.

The processes of estimating modifiability and consequences, and regulating attention, are naturally influenced by expectancies. Positive expectancies (optimism) and negative expectancies (pessimism) are particularly relevant as optimists have been observed to engage in more active coping strategies (than pessimists) while, for example, adapting to breast cancer [11] and recovering from coronary artery bypass surgery [12]. This active-coping ten- 
dency may explain why optimists recover faster and have better outcomes following coronary artery bypass surgery [11], demonstrate greater adherence to health care recommendations and better outcomes after cardiac transplant [13] and engage in more health protective practices after testing positive for HIV [14]. Expectancies have powerful effects on appraisals of illness modifiability, as well. From the optimistic view, illness can be modified once the right method is found. Indeed, simply believing the right method has been found, reinforces optimism and, in doing so, may be healthy, in and of itself.

Once again, finding the right method is associated with the constructs of response efficacy, i.e. finding the methods or tools for making positive changes and self-efficacy i.e., using the tools effectively [8]. In the weighing of evidence related to response and self efficacy, the patient's ability to sustain critical examination may become more evident, as some patients are highly susceptible to emotionally-driven (versus rational) treatment selection, and prone to emphasizing what other substances and individuals can do (response efficacy) rather than what they can do, themselves (self efficacy).

The six model factors are best understood as dynamically interacting, each potentially influencing others. For example, patients who estimate a low likelihood of modifying an intolerable condition through conventional medicine, may have needs for complementary therapies that supply a target for optimistic expectancies and, simultaneously, a way of reinforcing them. Under such conditions, they may either become highly critical in their selection of a complementary treatment, evincing a high level of attention regulation, or alternatively, highly uncritical, manifesting gaps in and breakdowns of regulated attention. In either case, an intolerable and worsening condition may reduce the need to minimize potentially negative consequences, because the consequences of not modifying the condition are so high.

Along these lines, we may see how the weighing of evidence can play a major or minor role in selection, depending on the level of attention regulation and the need to maintain optimistic expectancy. It is important to remember that evidence not only establishes a level of effectiveness, it establishes a range. If the patient needs a radical and unlikely change, he or she may actually avoid therapies with established ranges, opting for therapies which have not been tested, as these are more readily imagined as providing the efficacy required.

It may be evident by now that our model is nonlinear, although conveniently described in a linear manner. Changes in attention regulation may or may not precede changes in perceived consequences or modifiability or expectancies, just as changes in perceptions of response and self-efficacy may activate or be activated by changes in other factors. Rather than linear chain reactions, the model identifies feedback loops that evolve through positive (deviation-amplifying) or negative feedback (deviation-diminishing) patterns. In a given situation, either pattern may be adaptive, as the patient tries to adapt in the ways judged most relevant, rejecting activities judged ineffectual or too risky.

\section{Using the model to help the patient}

The test of guiding models is their capacity to identify factors that, in combination, cohesively explain behaviour. As this model guides our support program with cancer patients, we apply it clinically, testing and refining its utility in treatment provision and its validity in experimental studies. In trying to understand the motivations of patients seeking complementary treatments, how can it help?

\subsection{The quality of seeking - how you get there may be as important as where you get to}

Complementary therapy-seeking is predictable in circumstances where illness consequences are high and beliefs in the effectiveness of conventional treatments (i.e. response efficacy) are low. The model presented emphasizes that the patient's basic need for optimism about modification is healthy, and thus helps us understand their readiness to dis-regulate attention in order to sustain optimism. We are reminded (by placebo research) that optimism, itself, can be healing, even when attention regulation is compromised. As such, as practitioners, it is appropriate to caretake our patient's optimism, given that 
existing evidence suggests a balance towards optimism is healthier, when consequences are not too costly (in terms of risk exposure, out-of-pocket costs, social effects, disruptions of evidence-based treatment etc.).

However, in assisting our patients' health-seeking, we are also obligated to assess their attention regulation. In other words, we must help patients sustain a level of attention regulation and optimism that contributes to intelligent treatment selection, as well as overall functioning and quality of life. Along these lines it is important to take into account the findings on effective and ineffective attentional modes, observed in people confronting trauma and illness $[15,16]$. A curvilinear relationship emerges [15], with the extremes of dysfunctional attention characterized by distractability-avoidance, on one side, and intrusiveness on the other. Under extreme stress, individuals avoid focus on distressing events, while experiencing the unwelcome and chaotic intrusions of distressing thoughts. Under such conditions, poor decision-making is likely, if not inevitable. Thus we must help patients overcome dysfunctional attentional states, especially when observing their manifestation in complementary therapy seeking. Often the most effective way to do this is to inquire about the patient's own view of how their attention becomes disrupted to gauge the patient's awareness of these disruptions. If a patient is aware, according to the literature, he or she is likely to report an intrusive or avoidant pattern (or both). If either pattern is acknowledged, the patient may then be motivated to undertake some form of training in anxiety reduction or stress management (or accept a pharmacological treatment) to reduce the underlying emotional strain disrupting their attention regulation.

\subsection{Identifying when the seeking and use of complementary treatment signals the need for more in-depth counseling and psychotherapeutic support}

Beyond anxiety and stress reduction, we must be prepared to see needs for more in-depth, illnessadaptation counseling when examining the complementary therapy seeking of patients. The most obvious case is when the patient fails to use a highly effective evidence-based treatment (even though the consequences of not using it are probable and severe) because a high optimistic expectancy (accompanied by a minimum of critical examination) is placed on an complementary therapy with weak supporting evidence. In such a situation we may expect to find, according to the model, relatively low levels of attention regulation accompanied by high levels of anxiety and negative affect (masked or unmasked). Under such conditions, complementary therapies may be fastened onto as a convenient way of avoiding confrontations with more disturbing realities, such as the distressing or debilitating side effects that may be associated with an evidencebased treatment. The problem may not be fixed, however, if a switch to the evidence-based treatment is achieved. Unresolved disease adaptation issues may still be avoided and may require further assistance, in a counseling context, to be resolved

\subsection{Envisioning the optimal therapy-seeking process}

The scenario of negative "complementary" therapy seeking raises the question of what characteristics might be expected in an optimal process. When envisioning this, we begin to consider the types of empirical data that must be collected on "complementary therapies" to better equip us to counsel patients. What might an optimal mode of health seeking look like? According to our model, we might see both evidence-based and complementary therapies selected on the basis of their high probability of effectively modifying the disease condition. Furthermore, optimal treatment selection might include a detailed weighing of the pros and cons of the consequences of use, including the likely experience of using the treatments, as well as the likely consequences of using no treatments or other alternatives. It is expected that these considerations would not just be driven by optimistic expectancies, but by a "realistic optimism" that involves a nonavoidant examination of treatments. Lastly, we might expect that consideration be given to the patient's own self efficacy (their ability to maximize the success of treatment through their own actions) as well as the use of the best available agents and expertise in altering disease processes and consequences.

In conclusion, we have presented a model of 
health-seeking that can lead to better understandings of the complementary therapy seeking of our patients, and more insight into their disease adaptation process overall. From the perspective of this model and others constructed for similar purposes, studying the seeking of complementary therapies provides a window into the real life experience of our patients, as they confront diseases with different consequences and potentials for modification. We are clearly obligated to assist in their evidence-based and complementary therapy seeking and to achieve an indepth understanding not only of their disease, but how it affects them psychologically. This as always, is an empathic as well as theoretical challenge.

\section{References}

[1] Ritvo P. Quality of life and Prostate Cancer Treatment: Decision-Making and Rehabilitative Support. Canadian Journal of Oncology 1994;Suppl 1:S43-46.

[2] Ritvo P, Irvine J, Katz J, Shaw BF. Cognitive Group Therapy with Medical Patients. In: Farmer F, White J (Ed.) Cognitive group therapy. Washington, DC: American Psychological Association Books. (in press)

[3] Irvine MJ, Ritvo PG, Katz J, Shaw BF. Maximizing adherence with non-pharmacological treatment. Canadian Journal of Cardiology 1994;Pfizer Suppl:6-8.

[4] Katz J, Ritvo P, Irvine MJ, Jackson M. Coping with chronic pain. In: Zeidner M, Endler NS, editors, Handbook of coping, New York: Wiley, 1996.

[5] Ajzen I, Fishbein M. Understanding attitude and predicting behavior, Englewood Cliffs, NJ: Prentice Hall, 1980.

[6] Maddux JE, Norton LW, Stoltenberg CD. Self-efficacy expectancy, and outcome: value relative effects on be- havioral intentions. Journal of Personality and Social Psychology 1986;51:783-9.

[7] Weinstein ND. The precaution adoption process. Health Psychology 1988;7:355-86.

[8] Bandura A. Self-efficacy: toward a unifying theory of behavioral change. Psychological Review 1977;84:191-215.

[9] Prochaska JO. Strong and weak principles for progressing from precontemplation to action based on twelve problem behaviors. Health Psychology 1994;13:47-51.

[10] Prochaska JO, DiClemente CC, Velicer WF, Rossi JS Standardized, individualized, interactive, and personalized self-help programs for smoking cessation. Health Psychology 1993;12:399-405.

[11] Carver CS, Pozo C, Harris SD, Noriega V, Scheier MF, Robinson DS, Ketcham AS, Moffat FL, Clark KC. How coping mediates the effect of optimism on distress: a study of women with early stage breast cancer. Journal of Personality and Social Psychology, 1993;65:375-90.

[12] Scheier MF, Magovern Sr GJ, Abbott RA, Matthews KA, Owens JF, Lefebvre RC, Carver CS. Dispositional optimism and recovery from coronary artery bypass surgery: the beneficial effects on physical and psychological well-being. Journal of Personality and Social Psychology 1989;57:102440.

[13] Leedham B, Meyerowitz BE, Muirhead J, Frist WH. Positive expectations predict health after heart transplantation. Health Psychology 1995;14:74-9.

[14] Scheier MF, Carver CS. Dispositional optimism and physical well-being: the influence of generalized outcome expectancies on health. Journal of Personality 1987;55:169-210.

[15] Horowitz M, Wilner N, Alvarez W. Impact of event scale: a measure of subjective stress. Psychosomatic Medicine 1979;41:209-18.

[16] Bremner JD, Scott TM, Delaney RC, Southwick SM, Mason JW, Johnson DR, Innis RB, McCarthy G, Charney DS. Deficits in short-term memory in posttraumatic stress disorder. American Journal of Psychiatry 1993;150:1015-9. 